\title{
Cultural transposition of a thinking classroom: to conceive possible unthoughts in mathematical problem solving activity
}

\author{
Maria Mellone ${ }^{1} \cdot$ Tiziana Pacelli $^{1}$ (D) $\cdot$ Peter Liljedahl $^{2}$
}

Accepted: 12 March 2021 / Published online: 3 April 2021

(c) The Author(s) 2021

\begin{abstract}
This study concerns a professional development course designed and implemented for prospective teachers, centred on a teaching method regarding problem-solving activity, namely, the Thinking Classroom. The study is framed in the theory of cultural transposition, a perspective about the encounter with teaching practices from different cultural/school contexts. Cultural aspects are considered crucial and this encounter between cultures is seen as an opportunity for actors to become aware of their own unthoughts, i.e., some of the 'invisible' cultural beliefs about teaching and learning absorbed by their own culture. According to this framework, we present the results from a questionnaire given to all the participants, and two case studies of prospective teachers involved in the professional development, in order to discuss the kind of unthoughts on which they have focused in thinking about this training experience.
\end{abstract}

Keywords Cultural transposition $\cdot$ Thinking classroom $\cdot$ Unthoughts $\cdot$ Mathematical problem solving

\section{Introduction}

How is the term 'problem' defined? In Chinese, it can be expressed with the ideogram 问题 [Wèntí] that means question, challenge, referring also to the pleasure derived from this process. However, in the Western part of the world, this word $^{1}$ is often understood to indicate difficulty. Many different nuances of meanings and feelings associated with the idea of problem solving, as well as different strategies and representations used by students to solve mathematical problems, are dispersed through different countries and cultures (e.g., Cai, 2004). In recent years, some scholars have started to examine how students' ideas and attitudes-meant as a mix of emotions, beliefs, and perceived competences on mathematical problem - are related to the lived school experience (e.g., Di Martino, 2019). Empirical findings indicate that these factors can strongly influence students' approaches to problem solving (Di Martino \& Zan, 2011). The ideas that mathematics teachers have of mathematics

Tiziana Pacelli

tiziana.pacelli@unina.it

1 Department of Mathematics, Università degli studi di Napoli Federico II, Naples, Italy

2 Faculty of Education, Simon Fraser University, Burnaby, Canada and problem solving are also strongly conditioned by their personal/professional experiences (Berk \& Cai, 2019) and by their cultural context. In turn, these mathematics teachers' ideas and attitudes shape their educational practices, thus influencing students' mathematics learning (Berk \& Cai, 2019). For this reason, we believe that it is interesting to build cross-cultural research experiences focused on different ways of looking at mathematics in general, and mathematical problem solving in particular.

In this study, we present an encounter of a group of Italian prospective teachers (PTs) with a different way of working with mathematical problem solving, coming from a different cultural context. In particular, we designed and implemented for them a professional development (PD) experience centred on the Thinking Classroom (TC) (Liljedahl, 2016, 2020). The study is situated in the larger context of teacher learning literature, including works on PD related to changes in beliefs, as effective PD should increase teachers' knowledge while reflecting on their beliefs, which should be reflected in their educational practices with the aim of improving student learning (Berk \& Cai, 2019; Cai et al 2019). Our aim was to ascertain if and how encountering

\footnotetext{
1 In Neo-Latin languages the word 'problem' comes from Latin 'próblema' and from Greek 'probállein' that means 'to display in front'.
} 
this new educational practice within the PD changed the PTs' idea of problem solving.

The PD and the study are framed in the Cultural Transposition perspective, according to which it is expected that when teachers are exposed to teaching practices from different cultures, they would become more aware of their own unthoughts (Jullien, 2006), defined as some of the 'invisible' cultural beliefs about teaching/learning that they have absorbed from their own culture. We tried to understand how being presented with a different problem-solving culture allowed PTs to develop new perspectives about solving mathematical problems. The encounter with the TC, in our opinion, can therefore represent an opportunity for PTs to confront and re-examine their cultural beliefs, to learn and try new teaching strategies, and to reflect on their experience in collaboration with other teachers (Berk \& Cai, 2019).

Hence, the research question guiding the study in this paper is as follows: Which kind of unthoughts on mathematical problem solving can PTs develop within a PD course framed in the Cultural Transposition framework and entailing the Thinking Classroom?

\section{Cultural transposition}

Cultural Transposition is a perspective framing the use of mathematics educational practices adopted in other cultural contexts as an opportunity for questioning the didactic practices of one's own cultural context, in order to reconsider the educational intentionality underlying all educational praxis. It involves researchers and teacher-researchers in mathematics education who encounter teaching practices from other cultures and subject those to a deconstruction process in order to re-interpret their components through the lens of the beliefs and values of their own culture. Deconstruction is defined as "an analysis of the different levels in which a culture is stratified" (Derrida, 2002, p.1). ${ }^{2}$ It is done with a dual intent, i.e., to make the new teaching practice compatible with the beliefs, customs, and values of one's own cultural context, and to maintain features that are not usually associated with one's own cultural context. We believe, in fact, that the transposed teaching practice should embed some of the cultural aspects of its original context, as this would allow teachers to come into contact with different values and beliefs on mathematics teaching/learning.

In this perspective, the brutal import-export practice of educational methods is seen not only as failing but also dangerous. Exposure to mathematical practices that come from different cultures, if suitably managed, can create spaces of

\footnotetext{
2 http://ricerca.repubblica.it/repubblica/archivio/repubblica/2002/07/ 03/calma-scienzia-io-vi-rispetto.html.
}

reflection and awareness development for researchers, educators, and teachers. Thus, in adopting the Cultural Transposition perspective, the goal is not to perform a comparative study, but rather to open a dialogue between two different cultures in which every thought, in encountering the other culture, questions its own unthought (Jullien, 2006). The crucial idea of unthought conceived by Jullien can be related to the notion of cultural beliefs re-elaborated in mathematics education; it denotes the beliefs that people have unconsciously incorporated-within a certain degree of re-elaboration and personalisation-by living immersed in certain cultural contexts and which, for this reason, they take for granted until they encounter a different culture (Bartolini Bussi \& Funghi, 2019). In particular, we refer to the idea of culture suggested by D'Ambrosio (2010), as follows:

Upon recognizing that the individuals of a nation, community, or group shared their knowledge, such as language, systems of explanation, myths and spiritual gatherings, customs and culinary habits, and that their behaviours are made compatible with and subordinates to value systems agreed to by group, we say that these individuals pertain to a culture. In sharing knowledge and making behaviour compatible, the characteristics of a culture are synthesized. Thus we speak of the culture of the family, the tribe, the community, the association, the profession, the nation. (p. 10)

This view of culture allows us to argue that it is possible to recognize different cultural communities on the basis of the agreements and values systems, often not explicit, that the individuals in these communities share. In particular, school communities represent specific micro-cultures sharing institutional norms that often-remaining tacit—are not embraced with awareness or questioned. In this scenario, the encounter with educational practices referring to other values and regulatory systems can be a valuable resource in teacher training practices. The dialogue between different educational practices allows practitioners to become more aware of each teaching choice, by getting to know a different practice. The effectiveness of an educational method depends on how it is used and the micro-cultural context in which the people adopting it are immersed.

Consequently, teachers involved in PDs framed in Cultural Transposition are led to become aware of their unthoughts (Jullien, 2006), which are taken for granted and, in a sense, considered mandatory. One example of unthought emerged in a previous study as a part of which a group of Italian in-service primary teachers were exposed to the 'problems with variations' method, an educational tool used in Chinese primary schools that promotes early algebraic thinking. Encountering this method made the Italian teachers aware of the absence of attention to algebraic thinking in the Italian primary school practice 
(unthougth), and its importance in the early stages of mathematical instruction (Mellone et al. 2018).

\section{The thinking classroom and its deconstruction}

In previous work on Cultural Transposition, the deconstruction was developed from an outsider's point of view, i.e., not from the original culture from which the particular educational method came (Mellone et al. 2018, 2020). In contrast, for this study, the deconstruction was implemented both by the Italian authors and PTs as outsiders and by adopting Liljedahl's inside perspective, given that he was the designer of the transposed educational method.

Liljedahl (2016) defined a Thinking Classroom as "a classroom that is not only conducive to thinking but also occasions thinking, a space that is inhabited by thinking individuals as well as individuals thinking collectively, learning together and constructing knowledge and understanding through activity and discussion" (p. 362).

His research on TC $(2020,2016)$ emerged out of the observation that in a typical Canadian classroom, students did very little thinking. In fact, he found that on average $20 \%$ of students spent less than $20 \%$ of their class time thinking while the remaining $80 \%$ spent almost no time thinking. He also realized that the institutionally normative structures that were born at the birth of public education at the end of the first industrial revolution, and which are still present in the twenty-first century, were complicit in not only enabling, but also promoting, this lack of thinking. This is not to say that education had not changed over the course of the last 150 years. But students were still sitting, and teachers were still standing, and teachers still wrote on the board while students wrote in their notebooks.

For many years he sought to disrupt these and other institutionally normative practices with the intention of seeing if such disruptions could increase the number of students who are thinking, as well as increase the amount of class time during which they are thinking. Out of this research emerged a set of 14 practices that radically transformed the institutionally normative classroom into classrooms in which thinking was not only facilitated, but also necessitated:

1. The type of tasks: Engaging, collaborative, and stimulating to get students talking to each other while including a rich mathematics.

2. How tasks are given: Orally, motivating groups to discuss the task focus rather than trying to decode written instructions. Moreover, tasks have to be immersed in telling a story.
3. How groups are formed: Visibly at random, in order to remove social barriers, and to increase the mobility of knowledge and the enthusiasm for mathematics.

4. Student workspace: Vertical non-permanent surfaces (e.g., whiteboards or windows) in order to see the work done, with only one marker per group to stimulate discussion.

5. Room organisation: The teacher has to address the class from a variety of locations and use all the walls of the room, in which the desks are placed randomly.

6. How questions are answered: Only the so-called 'keepthinking' questions are addressed in order to allow students to return to work.

7. How hints and extensions are used: It is necessary to create a perfect balance between the challenge of the task and the ability involved in working on it, as students would otherwise get bored or frustrated.

8. Student autonomy: Teacher should encourage interaction among the groups.

9. When and how to level the class: Once a minimum threshold is passed, the teacher has to engage the groups in a discussion about the experience and understanding.

10. Student notes: Students should provide feedback about the work done by their team or other groups.

11. Practice questions: Questions should probe students' understanding.

12. Formative assessment: Focused on informing students about where they are and where they are going in their learning.

13. Summative assessment: Focused on the learning processes.

14. Reporting out: Focused on discerning the learning a student has demonstrated.

In the attempt to deconstruct the $\mathrm{TC}$ we have to recognize that amongst what has been observed in Canadian school communities, the asymmetric position of the teacher in relation to the students' position-the so-called frontal class - that gives the possibility of controlling the student body, has appeared impressive. This scenario seems to evoke the idea of a surveillance position of bodies and minds, described by Foucault (1975) in connection with prisons, military institutions and factories, and which somehow expresses, in its organization, the panoptic desire of modern Western societies. On the contrary, in the TC organisation, it is possible to recognise the desire for a break with this organization, with the school routine, and also with the usual way of working with mathematics. Indeed, it provides a suitable educational space in which each student is encouraged to stand up and get actively involved in mathematical problem solving in small groups. 
The Italian cultural/school context is still affected by some historical events that took place at the beginning of the last century, after the First World War and at the beginning of Fascism. Giovanni Gentile, a supporter of a humanistic culture, signed the school reform with a consequent downgrading of mathematics in the cultural development of the country. Moreover, the Italian cultural context is also deeply influenced by the presence of the Vatican State within its territory. The majority of the population is Catholic, which is relevant for our study because it helps us to recognise that the organisation of the interior space of the churches, with the frontal arrangement of the altar and the pews facing it, is an important cultural feature that influences the sense of belonging, which is emulated in the classrooms by placing the teacher's desk in front of those occupied by pupils.

Thus, the deconstruction process conceived in this experience of Cultural Transposition allowed us to recognise that the culture of teaching in Italian school communities is very different from the one proposed for the Thinking Classroom. The shared institutional norms are linked with a very rigid classroom organisation, in which pupils sit in desks arranged in parallel rows and in front of the teacher's desk. There is no tradition of specific care in the design of mathematics lessons, based mainly on the teacher's theoretical explanations and the execution of stereotyped exercises by students.

\section{Methodology}

In this section, we first present the design of a PD for PTs framed in the Cultural Transposition perspective and focused on the TC. Next, we describe the methods we chose to collect and analyse the data for inquiring into which kind of unthoughts on mathematical problem solving PTs develop in this PD.

\subsection{The educational device}

Since 2012, a group of Italian researchers has been designing and implementing PD courses framed in the Cultural Transposition perspective for in-service primary teachers in Reggio Emilia and Naples (e.g., Bartolini Bussi et al., 2017; Mellone et al., 2018, 2020). For this study, we experimented with something new, whereby we designed a PD for PTs focused on a particular mathematical educational practicethe Thinking Classroom.

The PD was implemented as part of the "Elementary Mathematics from a Higher Point of View' course taught to students pursuing the Master's Degree in Mathematics at the University 'Federico II' of Naples, Italy, and the course involved 25 PTs. It represented for the students the first opportunity to deepen mathematics education topics, such as problem solving and some methods used to teach mathematics. Generally, students wishing to become secondary school teachers choose this course, and for this reason we considered them PTs. Currently, in Italy, to be hired as a secondary school teacher with an open-ended contract, it is necessary to pass a selection process, in which one can participate after obtaining a Master's Degree in Mathematics and a certain number of educational credits in pedagogical and disciplinary subjects. Moreover, our PTs are supposed to have strong mathematical knowledge, as several courses in algebra, calculus, geometry, and physics must be completed to acquire the Bachelor's Degree in Mathematics.

The PD was designed and implemented in three phases, namely immersion in intensive TC workshops, participation in a TC deconstruction process led by an educator, and TC design/implementation by the PTs for the colleagues in the course.

In Phase 1, the PTs participated in four TC workshops given by Liljedahl, each lasting about two hours, during which they were involved in problem-solving activities of different levels of difficulty, some of which were chosen by Liljedahl from a collection of 'good problems' on his website. ${ }^{3}$ The TC was carried out considering points 1-9 in the teaching practices list reported in Sect. 3. Points 10-14 were not implemented because the aim was to prompt the PTs to reflect on both the proposed tasks and the teaching method they were experiencing. Before arriving at the workshops, the PTs received no information about the TC. Hence, only in Phase 2, a TC deconstruction occurred. Some effort was made to set up the space, as the Italian university lecture rooms are not always suitable for TC, given that, for example, the chairs and desks are fixed to the floor. Thus, they were carefully equipped with tools purchased for the occasion, including transparent plastic sheets that served as vertical non-permanent surfaces along all the walls of the room, and socks to be used to wipe them clean.

In Phase 2, the PTs were guided in the deconstruction process by an educator. During this 2-h ad-hoc reflection session, experiences lived in the Phase 1 were discussed in the light of the observations presented in Sect. 3. In particular, the deconstruction juxtaposed the PTs' TC experience with their experiences as students in Italian classrooms. At the end of this phase, PTs were instructed to read the paper published by Liljedahl (2016).

In Phase 3, PTs were asked to design and implement a TC for their colleagues with the potential of applying it in a secondary school classroom. Unlike the previous experiences of $\mathrm{PD}$ in the Cultural Transposition perspective for in-service teachers (Bartolini Bussi et al., 2017; Mellone et al., 2018, 2020), these PTs did not really work in a school. Thus, we wanted to create the conditions for them to experience the

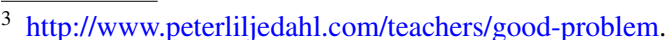


design and the leading of a TC. They were given the freedom to choose the mathematical content and the tasks to be proposed, with the possibility of consulting the collection of 'good problems' on Liljedahl's website. We asked the PTs to work in pairs to give them the opportunity to challenge their cultural beliefs by designing and managing a different class from those they were used to (Berk \& Cai, 2019; Cai et al., 2019).

\subsection{Research device}

\subsubsection{Data collection}

Three types of data were collected, the first of which was obtained through a written questionnaire that all PTs completed after Phase 1, aiming to capture initial reflections on their experiences of immersion in the TC as a new teaching practice. They were asked the following questions:

Q1) What did you like about the Thinking Classroom?

Q2) What didn't you like about the Thinking Classroom?

Q3) What could the educator have done to make it less engaging?

Q4) What could the educator have done to make it more engaging?

Q5) Which task did you like the most? Why? Try describing the text and the main resolution steps (and/or generalisation).

Q6) What do you think are the 'elementary from a higher point of view' mathematical aspects of the task?

Q7) Would you apply the Thinking Classroom at school? When, how, and why?

The second type of data included the notes taken by the educator during the direct observation of the PTs' TC implementation for their colleagues, along with other materials provided by the PTs. The latter were given to the class after the TC implementation in order to leave a written trace of the mathematical task given orally during the TC, as well as to provide further information on the chosen mathematical content.

The third type of data was obtained via individual semistructured interviews-recorded and transcribed-conducted nine months after the PD. Although several PTs took part in the interviews, here we present only two case studies. All interviews were guided by the Elicitation Interview protocol (Vermersch, 1994), conceived in the more general context of the social sciences, but also used in mathematics education (e.g., Coppola et al., 2019). It is based on techniques for the formulation of re-launchings, aimed at facilitating a-posteriori verbalisation to elicit detailed information about a particular lived experience. Only the following brief hints were prepared in advance, allowing the interviews to progress in line with the answers received (Cesari Lusso et al., 2015):

- When you were a school student did your teacher propose problems to you? Would you like to tell me what you remember?-focused on the interviewees' experience as school students;

- You attended TC workshops. Would you like to tell me what you remember? At one point you were asked to prepare a Thinking Classroom. Would you like to tell me what you remember?-focused on the TC immersion and how the TC design/implementation was done for colleagues;

- Now imagine you are a high school teacher. Would you implement the Thinking Classroom? How come?-the aim here was to probe into the PT's educational intentionality as a future in-service teacher.

We believe that these three complementary data types helped us answer our research question more completely. The questionnaire probed into the aspects of the TC that the PTs liked/disliked, on the PTs' engagement, on which mathematical tasks and content PTs were reflecting, and on how the PTs' intended to use the TC in the future at school. We expected that these questions would reveal some 'seeds' of the unthoughts on mathematical problem solving absorbed by their culture. The aim of the data collected through direct observation of TC implementation and via additional resources was to gain an understanding of how the seeds of these unthoughts, prompted by Phase 1 of the PD-and described in the questionnaire-affected the design of a TCbased mathematics lesson. Finally, the aim of the elicitation interview was to establish which kinds of unthoughts on mathematical problem solving the PTs could develop, with respect to the past, after experiencing a $\mathrm{TC}$ in the present and, therefore, which changes could occur in their intentional educational practice in the future.

\subsubsection{Data analysis}

The analysis of the data from questionnaire and interviews commenced with very careful reading of all materials. First, two of the authors separately interpreted the PTs' responses before comparing their findings. This was followed by a more analytical content analysis (Berelson, 1952; Blanchet, 1985), which involved classifying the lexical units related to the same topic, and segmenting the data into semantic units. Initially, an analysis of the lexical units frequencies was carried out, allowing some semantic categories-units of analysis having the same meaning or similar meaningsto emerge. Then, the frequencies of the lexical units in these categories were evaluated. This resulted in three main themes, that is, those with a higher frequency of lexical units 
related to the same topic, namely, affect (statements linked to the positive/negative emotions), task and mathematical content (statements regarding mathematical aspects involved), and method (reflections on teaching methods).

Later, we conducted a further analysis of the elicitation interviews in order to reflect on the PTs' Past, Present, and Intentional Future. In particular, for each main theme (affect, task and mathematical content, and method), by using the content analysis procedure, we identified the following:

- The unthoughts on mathematical problem solvingabsorbed by the culture in the Past: obtained as a grouping of lexical units referring to the same cultural beliefs by examining the narration of the past experience as school students.

- The causes that provoked the unthoughts-after TC experience in the Present: obtained as a grouping of lexical units referring to the same features of the TC, which, by the analysis of the report on TC immersion, seemed to have impressed PTs.

- The changes in PTs' educational practice-in the Intentional Future: obtained as a grouping of lexical units referring to the same aspects of educational practice, which emerged through the analysis of the reports of TC's design/implementation and via the elicitation of the educational intentionality as future in-service teachers.

In the sections that follow, after presenting the results yielded by the analysis of the questionnaire responses provided by all PTs, we focus on the three types of data related to two of the PTs (we use the pseudonyms Vincenzo and Marco) who participated in all three PD phases and designed/implemented a TC jointly. The choice to present the case studies of these two PTs was based on some of their features that made them particularly suitable for addressing our research question. First, they can be seen as typical examples of some other cases since, as was revealed by analysing their data, the sentiments they expressed were shared by the majority of the PTs, but they were capable of articulating them with particular clarity. Although these cases were seen as typical, they were not identical-there were some differences between them, which was also reflective of the majority of the PTs. First of all, Vincenzo often expressed his satisfaction with his past mathematical experiences; Marco, on the other hand, was not satisfied with his scholastic experience. From our point of view, this dissatisfaction made Marco more interested in teaching practices different from the ones he was used to, and Vincenzo's satisfaction caused him to be less willing to question the type of mathematical activity of his cultural context. Another significant difference was that Marco declared that teaching had always been his ambition since he was a child, while Vincenzo said that teaching mathematics was not his
Table 1 Number of PTs referring to the three themes

\begin{tabular}{ll}
\hline Theme & $\begin{array}{l}\text { Number } \\
\text { of PTs } \\
(\mathrm{n}=25)\end{array}$ \\
\hline Affect & 22 \\
$\begin{array}{l}\text { Task and math- } \\
\text { ematical content }\end{array}$ & 14 \\
Method & 23 \\
\hline
\end{tabular}

main future goal. Hence, we wanted to see what kind of unthoughts on mathematical problem solving, absorbed in the past according to their different cultural/school contexts, they could realise due to participation in the PD, and also by interacting with each other in the TC design/implementation.

\section{Results of analyses}

\subsection{The questionnaire}

Content analysis of the questionnaire responses (Table 1) revealed that 22 of the 25 participating PTs provided at least one answer that contained lexical units with the same or similar meaning referring to the theme affect. The most recurrent lexical unit was 'engagement' ${ }^{4}$ or a similar word or phrase, followed by 'stimulating', 'motivating', and 'not boring'. Whereas in Marco's answers there were not many references to the theme affect, in Vincenzo's ones there were explicit references to it, supported by the occurrence of lexical units such as 'appreciated' and 'pleasant'.

Regarding the theme method, the questionnaire analysis showed that almost all PTs (23 out of 25) explicitly referred to some features of the new educational practice in at least one answer. The most recurrent lexical units were 'groups' and 'space'. Vincenzo's answers concerned some of the 'advantages' that the new educational practice seemed to offer and the care that Liljedahl put into structuring the educational space. He listed all the 'novelties'-the term he chose- that he personally experienced in TC workshops, emphasising 'challenge without competition, random groups', stating that working standing next to a 'board' was 'much more pleasant' than using a sheet of paper. Also in Marco's answers there are references to working near the board, which 'stops being the teacher's space and becomes a space for common thinking'.

As can be seen from Table 1, only 14 PTs provided answers in which there was at least one lexical unit related to the theme task and mathematical content. Not everyone made explicit references to the structure of the tasks, and very few participants mentioned 'narrative context' or the

\footnotetext{
${ }^{4}$ We report the lexical units using inverted commas.
} 
Fig. 1 PTs' answers to Q2

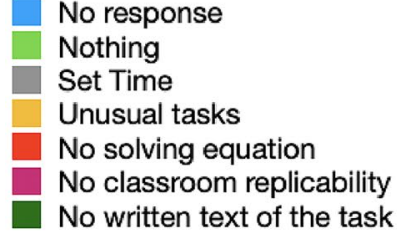

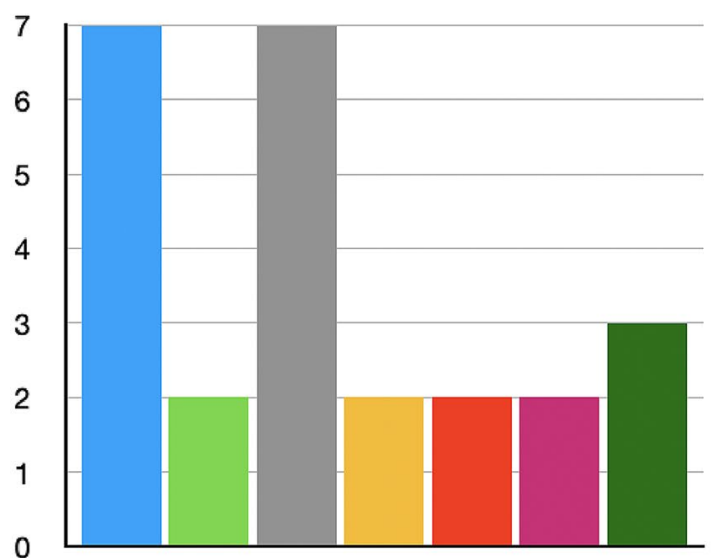

Q2 'text'. Moreover, only 7 of these 14 PTs highlighted that the tasks allowed them to proceed step by step until they could reach a generalisation. Vincenzo provided elaborate answers to Q5), showing, instead, how he was impressed by this aspect:

Q5) The task of the 25, because I noticed the transition from basic mathematics to much higher mathematics (logarithms, function, function study, etc). Text: Given the number 25, determine the positive numbers whose sum is 25 and whose product is as large as possible.

Solution: After moving from integers to rationals, to integer powers, to rationals with rational powers, we moved on to the reals and the writing the function $f(x)=x^{25 / x}$ which has a maximum in $x=e$ and models the situation.

The only reference to content in Marco's answers concerns tasks as a means of introducing new mathematical content and not as a means of practising mathematical content already introduced.

It should be noted that all PTs stated their intention to use TC in their future mathematics classes $(100 \%$ answered 'Yes' to Q7)). In particular, Vincenzo stated: 'Yes, I would. Of course, I'd need both physical and content tools, but I'd love to. But I'd still make a connection to the way we teach'. Vincenzo was clearly starting to think about how he could combine the TC 'novelties' with the way he knew how to teach, absorbed in his own culture, and how he could structure the task.

At the same time, if we look at Fig. 1 we can observe that some PTs-answering question Q2)—also state some TC aspects-regarding both the themes task and mathematical content, and method-that they did not like. In particular, in analysing PTs answers the following 7 separate categories emerged:
- No response: 7 PTs did not respond;

- Nothing: 2 PTs liked everything;

- Set time: 7 PTs would have preferred to have more time to solve the task-and not a set time;

- Unusual tasks: 2 PTs questioned the choice of non-typical tasks;

- No solving equation: 2 PTs did not like that a unique solving equation for the task is not provided at the end of the $\mathrm{TC}$;

- No classroom replicability: 2 PTs questioned the replicability of the TC in a real classroom;

- No written text of the task: 3 PTs would have preferred to have a written text of the task. Marco, for example, stated that: 'the absence of a written task may cause ambiguities in the understanding of the problem: maybe the two modes could be combined'.

The questionnaire analysis indicates that in the PTs some unthoughts related to affect, task and mathematical content, and method were germinating. For instance, the use of the word 'novelties' indicates Vincenzo's implicit reflection on his familiar educational practice. Similarly, Marco's observation on the transformation of the board from teacher's space to collective thinking space indicates an emerging awareness of his familiar school context. Moreover, Vincenzo's comment in his response to Q5) about the task moving from basic to higher mathematics is an indicator that this is unusual for him, as also for other PTs. For example, one of them, referring to the same task described by Vincenzo, stated: 'through this task we were able to see so much maths in just $1 \mathrm{~h}$, which without TC would have taken months!'. 


\subsection{Vincenzo and Marco's TC design/ implementation}

During their joint TC design/implementation, Vincenzo and Marco chose to use two tasks inspired by the example on palindrome numbers given on Liljedahl's website, and to introduce them through the telling of a story: a child, Anna, discovers a mirror that allows her to talk with her reflected image. She can access the mirror world by pronouncing different palindrome numbers every day. Once the 2-digit numbers have been used up, Anna asks herself how many days she will be able to continue using the 3 -digit numbers. So, the first task consists in finding out the number of 3-digit palindromes, followed by continued investigation looking for a general law regarding the number of $k$-digit palindromes.

Then Marco proposed the second task consisting in finding out how many 2-digit palindromic numbers of depth $k$ there are:

Anna's reflected images gives her another key to access her world of games: 'Go to the mirror and use the number 18 by rotating the mirror once, or use the number 37 by rotating it twice'. Anna asks her: 'How come these numbers?'. 'Easy, because $18+81=99$ which is a palindrome, while with 37 it has to be done twice, i.e., $37+73=110$ and $110+011=121$ is a palindrome'. So, having taken a two-digit number, how many times will Anna have to turn the mirror to enter?

Step by step the tasks designed by Vincenzo and Marco allowed their colleagues to proceed by trial and error. In addition, their TC design/implementation made possible a discussion on a variety of mathematical topics, moving from basic mathematics-definition of a palindrome, number of palindromes with 2-digit numbers-to higher mathematics-general law concerning $k$-digit numbers up to the discussion of Proof of the Sheldon Conjecture (Pomerance \& Spicer, 2019).

Vincenzo and Marco arranged the class space according to the TC design. Marco gave numbered cards to his classmates to divide them randomly into groups of three after which Vincenzo told the first part of the story. The groups were then instructed to use the boards labelled by the same number they had on their cards when solving the problem. Vincenzo and Marco were very careful to provide only strategic hints (as they saw Liljedahl doing during Phase 1 of the PD) in order to maximise the engagement. For example, if a participant asked 'Is it correct?' one of them replied 'What do you think?' or, when a group reached the solution, they proposed that they explore it in a more generalised situation. After almost all working groups had reached a solution, Vincenzo explored the participants' problem-solving processes, moving to the blackboards, focusing on differences and similarities. Then, Marco told the second part of the story and the participants proceeded with the mathematical task in the same way.

Direct observation allowed the researchers to appreciate the extreme care that Vincenzo and Marco dedicated to the choice of tasks, the design of the story conveying the mathematical problem, as well as the organisation of the space and the management of participants' questions, always trying to keep the engagement level high and giving feedback. It was evident that the entire class was actively involved in the proposed TC for its entire 2-h duration.

\subsection{Vincenzo and Marco's elicitation interviews}

Vincenzo and Marco's questionnaire responses and the observation of their joint TC design/implementation were insufficient for the researchers to grasp fully the evolution of their ideas about problem solving. To access their unthoughts on problem solving, absorbed from their own culture and provoked by the PD, we needed a suitable research device. The elicitation interview-due to its nature and structure-helped us to achieve this aim. By means of the content analysis of their interview responses, for each theme-affect, task and mathematical content, and method - we identified the following:

- The unthoughts on the problem solving that they absorbed from their cultures in the Past-focusing on the narration of their experiences as students.

- The causes that provoked their unthoughts, after TC experience in the Present-focusing on the report of their conscious and unaware actions and cognitive processes during the lived experience (Cesari Lusso et al., 2015; Vermersch, 1994).

- The changes in the educational practice in their Intentional Future - considering the description of their TC design/implementation and how they imagined themselves as in-service teachers.

Below we report some key excerpts from the two interviews. As mentioned in Sect. 4.2, they were conducted separately, but when they referred to the same question, Vincenzo and Marco's responses are written one after the other to highlight similarities and differences.

\subsubsection{The past}

With regard to the theme affect, in many passages of the interviews concerning the narration of Vincenzo and Marco's experiences as school students, lexical units such as 'anxiety', 'annoyed' and 'boredom' often 
occur. For instance, in the following excerpts ${ }^{5}$ (Jefferson, 1985) when Vincenzo and Marco described the typical mathematical activities that their mathematics teachers assigned to them at school, Vincenzo claimed to have always had a good relationship with mathematics, but he had mixed feelings about long texts involving mathematical exercises with 'long calculations'. These provoked a 'sense of challenge', but also made him anxious because he was afraid to make mistakes:

I: When at school a problem was proposed (.) before starting to solve it (.) how did you feel?

V: As a student (.) I have always had quite a good relationship with the subject (.) sometimes maybe when there was a long text I said 'wow this is long' (.) because I already imagined that they were long calculations (.) but I was never afraid of not being able to do it (.) I'd just stand there and try to solve it (.) then maybe if I noticed that I was jamming (.) there was the sense of challenge or maybe a moment of anxiety for me and I thought 'wow I can't do it'.

Marco, after stating that his teacher at primary school had 'absolutely' no problem-solving approach, spoke as follows:

M: My experience at school was not at all like that of the $T C$ (.) if one only has to do calculations and there is no reason to do them (.) I get annoyed.

For the theme method, in Vincenzo and Marco's interviews, there are various lexical units referring to transmissive teaching methods, as indicated in the following excerpts:

\section{I: How did your teacher make you work?}

$\mathrm{V}$ : It was always the usual things (0.5) explanations (.) exercises (.) questions (0.5) always from the point of view of a (.) frontal teaching (.) maybe in the final years when we were a more compact class (.) we did more group work but usually not because (.) they tend to make noise (0.5) so sometimes teachers avoid them.

M: Now I am (.) disgusted by what I did at school (.) I was given the procedural throughout high school (.) zero sense-making in what I did (.) only calculations calculations calculations so many calculations (.) until

\footnotetext{
5 In transcribing the interviews, we used some of Jefferson's norms (1985), such as (0.5) to indicate the length of the pause in seconds, while (.) indicates shorter pauses $(<0.5 \mathrm{~s})$. We also use ' $\mathrm{I}$ ', ' $\mathrm{V}$ ' and ' $M$ ' to refer to the Interviewer, Vincenzo, and Marco, respectively.
}

I came to university (.) teaching for me was the frontal lecture.

Vincenzo's mathematics teachers preferred individual work. The use of 'usual' or 'usually' by Vincenzo implies that something unusual can exist, just as the use of 'now' by Marco highlights the beginning — for him —of a dialogue between two educational practices from different cultural/ school contexts. A perspective that differed from their past experiences was apparently being revealed, bringing out the 'frontal teaching' organisation of the class as an unthought.

With respect to the theme task and mathematical content, both Vincenzo and Marco described that, during individual activities, they were either given mainly geometric tasks, based on figures and formulas, without any demonstration activities. In particular, Vincenzo noted: For the seconddegree equation they gave me the formula (.) they made me read the demonstration maybe to understand how to get there (.) but they never tried to make me do it (0.5).

From Vincenzo and Marco's narration of their past experiences as school students some important unthoughts on problem solving emerge. The cultural beliefs absorbed from their cultures in the Past become explicit and visible. For instance, the exercises with calculations provoked anxiety and annoyance, mathematical activities were assigned by transmissive teaching methods and individual work, frontal organisation of the class was prevalent, and problem solving was based on mainly geometric tasks.

\subsubsection{The present}

In the description of the conscious and unaware actions (Vermersch, 1994) carried out by Vincenzo and Marco during the lived experience, the most recurring lexical units are 'engagement', and similar words or phrases, or 'enjoy' and similar words or phrases. Both the PTs commented on having fun when solving the mathematical tasks proposed by Liljedahl during Phase 1. Most importantly, they claimed to have felt very involved, as can be seen from the following excerpts:

I: When solving the tasks, or more generally, when attending the Thinking Classroom, how did you feel?

$\mathrm{V}$ : Very engaged (.) very engaged (.) I mean I was having fun also because (.) at the beginning it was a trial and error problem and so you can't feel discouraged by something that you can't prove because it's just a matter of trying until you find a solution (.) then after (0.5) there was the generalization but that was already made easy by the knowledge of the problem we were solving using particular examples (.) because giving us examples before (.) increasingly complex examples 
allowed us to have a deeper knowledge of the problem and then allowed us to make a generalisation.

M: Enjoyed enjoyed (.) he always made you feel useful (.) even when you weren't going where you were supposed to go (.) he always valued what you said (.) that's an incredible thing.

The repetition of the expression 'very engaged' and 'enjoyed' at the beginning of the excerpts highlights how they felt during the problem-solving activities. In addition, Vincenzo never felt 'discouraged' and Marco felt 'valued'.

The theme affect seems to be closely related to task and mathematical content as well as method. Both the PTs were particularly impressed by the method implemented during the TC. In line with their questionnaire responses, they explained that were impressed by Liljedahl's care in setting up the TC. They commented on the way he divided the participants into groups 'by a method of random distribution and by using playing cards', as well as by the oral communication of the task 'through a narration'. In particular, they found the vertical boards convenient to use.

In addition, Vincenzo and Marco appreciate the tasks and the related mathematical content, as we could already see in the previous excerpts. Vincenzo described tasks as 'trial and error problems' that, by providing 'increasingly complex examples' allowed students to 'have a deeper knowledge of the problem and to make a generalisation'a feature that already emerged during the analysis of his questionnaire responses. Similarly, Marco stated: Step by step he tried to get an idea of the various solving strategies and if he thought it necessary he would bring up the problem (0.5) when a level was reached (.) he gave you the next task until you reached a more general level. This is evident also in the following excerpts:

V: There was the task that you had to solve (.) once it was solved (.) you would call Peter and say 'Is this the solution?' (0.5) if it didn't work well (.) he said 'you can improve' (0.5) if we asked him if it was right (.) he didn't say it was right (.) he always asked us if it was right for us.

I: When he did this (.) how did you react?

$\mathrm{V}$ : When we weren't sure he said 'keep thinking' (.) when we were sure (.) he said 'ok ok' and gave us (.) another task (.) similar (.) but maybe a little bit more complex and then a small step forward (.) and this continued until you came to a more general or more complex solution where the problem had become more complex.

In this excerpt, Vincenzo reiterated his admiration for Liljedahl's way of giving feedback, supporting the gradual path towards a process of generalisation of the mathematical concepts involved, similarly to what Marco described:
M: When Peter was giving you a suggestion and you were telling him something wrong (.) he never said 'what you are saying is wrong' (.) he tries to give it a value to lead you to the solution.

From the analysis of Vincenzo and Marco's TC experiences in the Present, some causes provoking the unthoughts emerged, such as the following: fun and engagement in problem solving; care in setting up problem-solving activities; feedback importance; tasks solved by trial and error and by a step-by-step approach involving examination of increasingly complex aspects until reaching a generalisation.

\subsubsection{The intentional future}

In the analysis of Vincenzo and Marco's TC design/implementation for their colleagues, the same three closely intertwined themes emerged. First, in the theme affect, the most frequently recurring lexical units were 'happy', 'like', 'amused' and other expressions similar in meaning, as shown below:

I: At one point, you were asked to prepare (0.5) and then propose to the class a Thinking Classroom (.) when this request was made to you (.) how did you feel?

V: Honestly I was quite happy because it was something that I liked and which (.) I enjoyed doing (.) an innovative method that I didn't think could engage so much (0.5) honestly, I was very happy.

M: At first I was amused (.) I was also a bit intrigued.

I: How did you feel when you were conducting the Thinking Classroom?

V: $\quad$ A little anxious because (.) I hoped it worked (.) I had fun (.) at the end we had done it myself and Marco (.) so it was our project (.) conceived by us made by us (.) then a little anxiety because I hoped it worked (.) but seeing that it was working I still felt quite (.) excited (.) in the sense that I had done something that (.) was going well.

In these excerpts, Vincenzo claimed to have been happy and stimulated in planning the TC because he had personally experienced that it was an innovative method involving participants. In implementing it, Vincenzo reported having had anxiety, caused by the hope that everything would go well. Anxiety turned into excitement when, in actually conducting the TC, he felt that it was working well. Marco, as well as stating that he had been amused, also expressed his curiosity that a new educational practice was being implemented.

During the interviews, both Vincenzo and Marco also commented on the meticulous way used to prepare the TC and the care given both to the implementation of the method and to the choice of the task and mathematical content. They 
described in detail the procedure that was adopted in the elaboration of the task based on particular mathematical content, the palindrome numbers. In addition, they stated that they chose to immerse the task in a narrative context and eloquently justified their choice, as shown below:

I: Why did you choose to put the task in a narrative context?

$\mathrm{V}$ : Because it's important to think of a real context that could happen (.) so you have an interest in resolving the situation (.) so making up a story is still important to get people involved (.) they almost identified themselves with the problem.

$\mathrm{M}$ : Storytelling can also make people listen to the problem (.) so they are more motivated to solve it (.) there is always that impulse to say (.) 'let's see in the story what these accounts are for'.

It is fundamental to underline that, in some passages of Vincenzo and Marco's interviews, we can observe the meeting - and their reflection on it - between mathematics educational practices that come from different cultures. In the last part of the interviews, when they were asked to imagine being high school mathematics teachers, they responded as follows:

V: I would immediately consider the Thinking Classroom but (.) the only trouble is that obviously you should create a lot of special tasks (.) it would not be something that I would do alone (0.5) but I don't know at this moment for example (.) how to develop topics of a particular school year via the Thinking Classroom (0.5) I know you can do it (.) because Peter also said you can do it (0.5) but then it would be an excessive workload (.) because anyway it is not easy to write a good task.

M: There were some things done by Peter that even went as far as using logarithms, particular functions (.) the tasks need to be adapted to the context (.) but I wouldn't change anything about the method at all.

These excerpts show that Vincenzo and Marco recognised the didactic effectiveness of the TC, having experienced and studied it. In addition, they began to wonder how to carry out the TC in their contexts, integrating the advantages of the new practice in others that involved culturally different setting, where for example it seems necessary 'to develop topics of a particular school year'. They were also beginning to reflect not only on the 'novelties' observed by encountering the practice from a different culture, but also on some unthoughts on the usual educational practice with regard to their cultural context. This constructive cultural shock pushed them to consider changes to the educational practices regarding method, the task and its mathematical content in their Intentional Future with respect to those usually adopted in the educational practice they knew as students, as made explicit by Marco: If I had experienced TC when I was a student (.) I would have gone crazy over something like this (.) because that's what I missed.

Vincenzo and Marco were aware of their-and of Italian mathematics teachers' in general- 'trouble' when faced with developing a mathematical task suitable for the TC and changing old habits.

$\mathrm{V}: \quad$ I think the main thing is the fear of the unknown (.) in the sense that (.) until I try it I don't convince myself (.) also because being used to working in a certain way (.) the difficulty could be for the teachers (.) the different way to manage a class.

$\mathrm{M}$ : If one never tries however (.) then we would always have to do frontal lessons for the next thousand years (.) whoever wants to teach first must be willing to change (.) if a study arrives (.) if a method arrives (.) that you try on your skin (.) convinces you (0.5) at least try it.

It seems that, for Vincenzo and Marco, Phase 1 and Phase 3 of the PD-TC immersion and TC design/implementationhad a quite crucial role.

Development of awareness regarding changes to the educational practice (method and task and mathematical content) on problem solving in the Intentional Future is confirmed when Vincenzo and Marco responded to the last question:

\section{I: What has changed?}

$\mathrm{V}$ : The belief that the only way I could teach (.) was to explain how something works (.) when instead I can create a method to get them to the solution.

M: I have discovered that the way I have been taught mathematics (.) throughout my life (.) is completely improvable (.) this has certainly changed (.) you can explore a problem (.) everything has an extension (.) even if it seems trivial.

It seems that for Vincenzo the unthought that it is always necessary to explain the procedure based on 'how something works' had been dispelled. In Marco's statements, it is evident how encountering the TC led him to reflect on the educational practice absorbed in his cultural context in order to improve it. In Table 2, we report what emerged in each theme with respect to the elicitation interview timeline. 
Table 2 Unthoughts, causes and changes related to the 3 themes

\begin{tabular}{|c|c|c|c|}
\hline & Affect & Method & Task and mathematical content \\
\hline Unthoughts in the past & $\begin{array}{l}\text { Exercises with calculations pro- } \\
\text { voke anxiety and annoy }\end{array}$ & $\begin{array}{l}\text { Mathematical activities by trans- } \\
\text { missive teaching methods } \\
\text { Individual work } \\
\text { Frontal organisation of the class }\end{array}$ & $\begin{array}{l}\text { Infrequent problem solving } \\
\text { Geometric tasks }\end{array}$ \\
\hline Causes in the present & $\begin{array}{l}\text { Fun and engagement in problem } \\
\text { solving }\end{array}$ & $\begin{array}{l}\text { Care in setting up problem-solving } \\
\text { activities } \\
\text { Feedback importance }\end{array}$ & $\begin{array}{l}\text { Tasks solved by a step-by-step } \\
\text { approach involving examination } \\
\text { of increasingly complex aspects } \\
\text { until reaching a generalisation }\end{array}$ \\
\hline Changes in the intentional future & $\begin{array}{l}\text { Happiness, motivation, curiosity } \\
\text { Anxiety turning into excitement }\end{array}$ & $\begin{array}{l}\text { Care of the educational space } \\
\text { Use of a well-structured narrative } \\
\text { context }\end{array}$ & $\begin{array}{l}\text { Choice of the content and task in a } \\
\text { meticulous way }\end{array}$ \\
\hline
\end{tabular}

\section{Concluding remarks}

The aim of this study was to elucidate which kinds of unthoughts on problem solving PTs can develop within a PD framed in the Cultural Transposition perspective (Mellone et al., 2018, 2020) and involving the TC (Liljedahl 2016, 2020). The PD was designed and implemented along three phases, comprised of immersion in TC workshops, participation in a TC deconstruction process, and TC design/ implementation for the class. We have presented the findings yielded by the analysis of the questionnaire responses provided by all 25 PTs that took part in the PD and the findings obtained by analysing three types of data (questionnaire, direct observation of a TC design/implementation, and elicitation interview) of two case studies involving Vincenzo and Marco-PTs who attended all the three PD phases and designed/implemented jointly a TC.

The content analysis of the questionnaire data shows that Phase 1 of the PD (TC immersion) already provoked in our PTs some reflections linked to three main themes-affect, method, and task and mathematical content - on the comparison between educational practices from different cultural contexts, namely, TC and the usual educational practice to which they were accustomed. Some unthoughts germinated; for instance, we noted in Vincenzo reflections on the 'novelties' of the TC not present in his familiar educational practice, and on tasks moving from basic to higher mathematics, unusual for him. In Marco, we observed a comment on the tasks as a means of introducing new mathematical content and not as a means of practising mathematical content already introduced, and a remark concerning the board becoming a space for thinking collectively, in contrast to the idea of it as the teacher's space, absorbed in his familiar school context.

On the other hand some TC aspects that the PTs disliked also emerged: set time, no written text of the task, the choice of some unusual tasks, not providing a solving equation. There are some PTs who probably still feel comfortable with the traditional Italian approach to teaching mathematics and would like to be more guided in the solving process, also questioning the replicability of the TC in Italian school communities. Marco himself doubted the effectiveness of an orally communicated task over a written text. It should be noted that in their TC implementation (Phase 3) Vincenzo and Marco decided to communicate the task orally, but then to provide their colleagues also with a written text. In our opinion, this is a clear example of a dialogue between two different cultures in which every thought, encountering the other culture, questions its own unthought (Jullien, 2006). In the educational intentionality of Vincenzo and Marco, the emergence of a 'give and take' between the two cultures that have met is evident.

Moreover, the analysis of the elicitation interviews allowed us to identify for each of the three themes some unthoughts on problem solving that Vincenzo and Marco absorbed from their cultural/school contexts, as follows: in the Past, the causes that provoked unthoughts, after their TC experiences in the Present; and the changes of the educational practice in their Intentional Future (Table 2). It seems that living the Present-Vincenzo and Marco's TC immersion within the PD-led them to reflect on what they experienced in the Past as school students, in order to push them to rethink how to implement in the Future mathematical problem-solving activities in a real classroom.

It should be noted that Vincenzo and Marco had different past school experiences and, at the beginning of the PD, while Vincenzo did not question the educational practices he was exposed to as a student, Marco was intrigued by new ones. In addition, they also declared different future job goals. But the analysis revealed some interesting points in common in the development of their idea of problem solving.

Indeed, the data show that Vincenzo and Marco's encounter with the TC-in the Present-has introduced them to new aspects of problem solving, e.g., fun and engagement in problem solving (affect), care in setting up problem-solving activities, feedback importance (method), tasks solved by trial and error and by a step-by-step approach involving 
examination of increasingly complex aspects until reaching a generalisation (task and mathematical content). Their knowledge of problem solving increased. These new features represent the causes of the emergence of some unthoughts on problem solving, which they had absorbed as school students in the Past, e.g., exercises with calculations provoke anxiety and annoy (affect), mathematical activities by transmissive teaching methods and individual work, frontal organisation of the class (method), infrequent problem solving based mainly on geometric tasks (task and mathematical content). It should be noted that, in the Italian National Guidelines for all school levels, references are frequently made to the development of problem-solving skills, but in the cultural/school context, in practice, teachers do not always spend enough time on these activities.

The TC experience in this PD provoked positive affect in Vincenzo and Marco-they felt 'happy', 'motivated' and 'intrigued' in planning/implementing a TC - and this is reflected in their Intentional Future, leading them to think about proposing the TC as teachers-as was the case for all PTs attending the PD. Moreover, the reflection on aspects of the Italian school system, during the TC deconstruction (Phase 2) allowed them to rethink both the method and the task and mathematical content they would like to implement. The data analysis from the direct observation of their TC implementation (Phase 3) and from their elicitation interviews shows that they took care when organising the educational space-unusual in the Italian cultural/school context, where classrooms are generally organised to facilitate 'frontal teaching' with a single blackboard - they used a well-structured narrative context, chose the mathematical content, and prepared the task in a meticulous way. In addition, they took care in the management of the participants' questions, trying to keep them engaged in the task. They gave great importance to the affective dimension in their problem-solving activity, which is often neglected in the cultural context of the Italian school and, instead, is emphasized in some other cultures, and in particular in Liljedahl's approach (2016).

Despite its limitations, this study highlights that the idea that mathematics teachers have of mathematics and hence of problem solving is conditioned by their personal/professional experiences (Berk \& Cai, 2019) and by the cultural context in which they live. Moreover, the 3-phase PD presented in this paper seems to be in line with some models concerning teachers' PD (Cai et al., 2019). According to their designers, in order to be effective, PD courses should increase knowledge, make teachers reflect on their beliefsincluding those derived from their cultural/school contextand lead to changes in educational practice, all with the aim of enhancing students' mathematics learning potential. The PD described here and elsewhere (Mellone et al., 2018, 2020), as a cultural activity encountering another culture
(D'Ambrosio, 2010), allowed our PTs (especially Vincenzo and Marco) to experience an educational practice from a different culture - the TC - to question the didactic practices of their own cultural context in order to reconsider the educational intentionality underlying all educational praxis.

It should be stressed that Vincenzo and Marco-as well as all the participants in the PD-were pre-service teachers and, therefore, certainly more ready for 'novelties' than in-service teachers. Moreover, during the Elementary Mathematics from a Higher Point of View' course, they had already discussed other new educational practices and reflected on different aspects of problem solving. In-service teachers would probably be more bound by the culture of the Italian school community in which they are deeply immersed and it would be more difficult to bring out their unthoughts.

Obviously, it would be interesting to conduct a study to understand how PTs, once they become teachers, will be driven by the unthoughts that emerged in their TC experience, in really constructing problem-solving activities with their students. But at the same time, this study should encourage researchers/educators in mathematics education to implement PD courses-based on encountering new educational practices from other cultures-both for pre-service and in-service teachers, aimed at supporting the emergence of unthoughts. They could represent valuable resources in teacher training practices.

Funding Open access funding provided by Università degli Studi di Napoli Federico II within the CRUI-CARE Agreement.

Open Access This article is licensed under a Creative Commons Attribution 4.0 International License, which permits use, sharing, adaptation, distribution and reproduction in any medium or format, as long as you give appropriate credit to the original author(s) and the source, provide a link to the Creative Commons licence, and indicate if changes were made. The images or other third party material in this article are included in the article's Creative Commons licence, unless indicated otherwise in a credit line to the material. If material is not included in the article's Creative Commons licence and your intended use is not permitted by statutory regulation or exceeds the permitted use, you will need to obtain permission directly from the copyright holder. To view a copy of this licence, visit http://creativecommons.org/licenses/by/4.0/.

\section{References}

Bartolini Bussi, M., Bertolini, C., Ramploud, A., \& Sun, X. (2017). Cultural transposition of Chinese lesson study to Italy: An exploratory study on fractions in a fourth-grade classroom. International Journal for Lesson and Learning Studies, 6(4), 380-395. https:// doi.org/10.1108/IJLLS-12-2016-0057

Bartolini Bussi, M. G. \& Funghi, S. (2019). Lesson study in primary pre-service teachers' education: Influences on beliefs about lesson planning and conduction. In M. Graven, H. Venkat, A. Essien \& P. Vale (Eds.). Proceedings of the 43rd conference of the 
international group for the psychology of mathematics education (Vol. 2, pp. 81-88). PME.

Berelson, B. (1952). Content analysis in communication research. Free Press.

Berk, D., \& Cai, J. (2019). Mathematics teacher beliefs. In M. A. Peters (Ed.), Encyclopedia of teacher education. Singapore: Springer. https://doi.org/10.1007/978-981-13-1179-6_236-1

Blanchet, A. (1985). L'entretien dans les sciences socials: l'écoute la parole et le sens. Bordas.

Cai, J. (2004). Why do U.S. and Chinese students think differently in mathematical problem solving? Exploring the impact of early algebra learning and teachers' beliefs. Journal of Mathematical Behavior, 23, 135-167.

Cai, J., Chen, T., Li, X., Xu, R., Zhang, S., Hu, Y., Zhang, L., \& Song, N. (2019). Exploring the impact of a problem-posing workshop on elementary school mathematics teachers' problem posing and lesson design. International Journal of Educational Research. https://doi.org/10.1016/j.ijer.2019.02.004

Cesari Lusso, V., Iannaccone, A., \& Mollo, M. (2015). Tacit knowledge and opaque action. In G. Marsico, R. Ruggieri, \& S. Salvatore (Eds.), The yearbook of idiographic science (Vol. 6, pp. 273-290). Information Age Publishing.

Coppola, C., Mollo, M., \& Pacelli, T. (2019). The worlds' game: Collective language manipulation as a space to develop logical abilities in a primary school classroom. European Journal of Psychology of Education, 34(4), 783-799. https://doi.org/10.1007/ s10212-018-0401-1

D'Ambrosio, U. (2010). Ethnomathematics—Link between traditions and modernity. Sense Publishers.

Derrida, J. (2002). In P. Odifreddi. calma scienzia io vi rispetto. La Repubblica. Orta, Italy, March 2002. http://ricerca.repubblica.it/ repubblica/archivio/repubblica/2002/07/03/calma-scienzia-io-virispetto.html

Di Martino, P. (2019). Pupils' view of problems: The evolution from kindergarten to the end of primary school. Educational Studies in Mathematics, 100, 291-307. https://doi.org/10.1007/ s10649-018-9850-3
Di Martino, P., \& Zan, R. (2011). Attitude towards mathematics: A bridge between beliefs and emotions. ZDM - The International Journal on Mathematics Education, 43(4), 471-483.

Foucault, M. (1975). Surveiller et punir. Paris: Gallimard. Italian version: Foucault, M. (2014). Sorvegliare e punire. Nascita della prigione (trans: Tarchetti, A.) Torino: Einaudi.

Jefferson, G. (1985). An exercise in the transcription and analysis of laughter. In T. Van Dijk (Ed.), Handbook of discourse analysis (Vol. 3). Academic Press.

Jullien, F. (2006). Si parler va sans dire. Du logos et d'autres ressources. Le Seuil.

Liljedahl, P. (2016). Building thinking classrooms: Conditions for problem solving. In P. Felmer, J. Kilpatrick, \& E. Pekhonen (Eds.), Posing and solving mathematical problems: Advances and new perspectives. New York: Springer.

Liljedahl, P. (2020). Building thinking classrooms in mathematics, grades $K$-12. Corwin Mathematics series. Sage Publications.

Mellone, M., Ramploud, A., \& Carotenuto, G. (2020). An experience of cultural transposition of the El'konin-Davydov curriculum. Educational Studies in Mathematics. https://doi.org/10.1007/ s10649-020-09942-7

Mellone, M., Ramploud, A., Di Paola, B., \& Martignone, F. (2018). Cultural transposition: Italian didactic experiences inspired by Chinese and Russian perspectives on whole number arithmetic. ZDM Mathematics Education, 51(1), 199-212.

Pomerance, C., \& Spicer, C. (2019). Proof of the Sheldon conjecture. The American Mathematical Monthly, 126(8), 688-698. https:// doi.org/10.1080/00029890.2019.1626672

Vermersch, P. (1994). L'entretien d'explicitation. ESF.

Publisher's Note Springer Nature remains neutral with regard to jurisdictional claims in published maps and institutional affiliations. 